\title{
Candidurie nosocomiali: analisi dei fattori di rischio, terapia ed evoluzione micologica. Risultati di una indagine nazionale
}

\author{
Elisabetta Faggi ${ }^{1,2}$, Gianluigi Lombardi ${ }^{1,3}$, Stefano Andreoni ${ }^{1,4}$, Esther Manso ${ }^{1,5}$, Paolo Fazii ${ }^{1,6}$, \\ Claudio Farina ${ }^{1,7}$, Gabriella Pini ${ }^{2}$, Gioconda Brigante ${ }^{3}$, Stefania Fracchiolla ${ }^{8}$, Mario Spinelli.', \\ Cristina Bonetti ${ }^{10}$, Anna Maria D'Accardo", Giorgio Verna ${ }^{5}$, Piero Anichini ${ }^{12}$
}

'Comitato Studio Micologia (CoSM), Associazione Microbiologi Clinici Italiani (AMCLI)

${ }^{2}$ Dipartimento Sanità Pubblica, sez Microbiologia - Università di Firenze

${ }^{3}$ Ospedale di Circolo e Università dell'Insubria - Varese

${ }^{4}$ A.O. Ospedale Maggiore della Carità - Novara

${ }^{5}$ A.O. Umberto I - Ancona

${ }^{6}$ P.O. Ospedale Spirito Santo - Pescara

${ }^{7}$ A.O. Ospedale San Carlo Borromeo, Milano

${ }^{8}$ A.O. SS. Annunziata - Taranto

${ }^{9}$ A.O. Ospedale S. Anna - Como

${ }^{10}$ Ospedale Maggiore - Crema

"Ospedale Cervello - Palermo

${ }^{12}$ A.O. Careggi - Firenze

\section{Nosocomial Candiduria: risk factors, therapy and outcome. Results of a nationwide survey}

Key words: Candiduria, Candidaemia, risk factors

\section{SUMMARY:}

The multiple problems associated with the recovery of yeasts from urine specimens induced the Medical Mycology Committee (CoSM) of AMCLI to run a nationwide epidemiologic survey on candiduria in order to evaluate risk factors, involved species, treatment and outcome.

Ten hospitals (Bergamo, Como, Crema, Novara, Varese, Ancona, Florence, Pescara, Palermo and Taranto) participated to this study, which was run on a 15-month period (October I, 2001-December 31, 2002). Overall, 83 Data Forms were collected, regarding patients admitted to Intensive Care Units (45), Surgical (9) and Medical (29) wards.

The most common risk factors were: bladder catheter, antibiotic therapy, parenteral nutrition, kidney failure, surgery. Candiduria, mostly asymptomatic, were often associated with fever and bacterial infections. Concurrent candidaemia was detected in 13 patients.

Candida albicans was the most frequently recovered species, from both urine and blood, followed by C. glabrata. Other Candida species were occasionally isolated from urine specimens.

Specific antifungal treatment was administered to $58 \%$ of the patients, mostly using fluconazole. The remaining subjects did not receive antimycotic therapy. Eradication of Candida from urine specimens was observed even without a specific therapy.

\section{INTRODUZIONE E SCOPO DELLA RICERCA}

L'isolamento dalle urine di lieviti del genere Candida è frequente nella popolazione ospedalizzata e la loro presenza può essere dovuta ad una banale contaminazione (da nicchie ecologiche dove il lievito è normalmente presente, quali - ad esempio - la vagina e il perineo), ad una transitoria colonizzazione o ad una infezione a carico dell'apparato urinario conseguente alla progressione del lievito dalle basse vie urinarie oppure ad una sua diffusione per via ematica in un quadro di candidosi disseminata $(2,7,9)$.

I molti interrogativi diagnostici e terapeutici posti dall'isolamento di lieviti dalle urine $(4,5,10)$ e soprattutto la possibilità che il loro ritrovamento possa essere indice di una candidosi profonda/disseminata stimolarono il Comitato Studio Micologia (CoSM) dell'AMCLI a proporre (3), a livello nazionale, un'indagine epidemiologica sulle candidurie allo scopo di indagarne i fattori di rischio, l'eziologia, l'approccio terapeutico, l'evoluzione micologica. L'indagine fu rivolta prevalentemente a pazienti degenti nei reparti di tera- 
pia intensiva in quanto maggiormente a rischio di sviluppare candiduria e candidosi sistemica.

\section{METODO DI INDAGINE E PARTECIPANTI}

L'indagine ha previsto la registrazione dei seguenti dati epidemiologici relativi a pazienti con almeno due urinocolture positive per lieviti (effettuate consecutivamente e con carica micotica $\geq 10^{4} \mathrm{UFC} / \mathrm{ml}$ ): età, sesso, fattori di rischio (catetere urinario, ostruzione vie urinarie, interventi chirurgici, terapie antibiotiche, terapie immunosoppressive, candidemia, candidosi orofaringea ecc.), sintomatologia (funguria asintomatica, disuria, stranguria, pollachiuria, leucociti e eritrociti nel sedimento ecc,), specie fungina isolata dalle urine e relativa concentrazione, specie fungina isolata dal sangue, approccio terapeutico (terapia antifungina, nessuna terapia antifungina, rimozione catetere urinario), evoluzione micologica (perdurare della candiduria, risoluzione) e la diagnosi conclusiva (micosi urinaria esclusa, probabile, accertata).

Hanno aderito all'iniziativa 10 centri ospedalieri distribuiti nel Nord (Bergamo, Como, Crema, Novara, Varese), nel Centro (Ancona, Firenze, Pescara), e nel Sud Italia (Palermo, Taranto).

L'indagine ha avuto la durata di 15 mesi (1 ottobre 2001 - 31 dicembre 2002).

\section{RISULTATI}

Sono state raccolte complessivamente 83 schede relative a pazienti ( 35 maschi e 48 femmine) ricoverati in reparti di Terapia Intensiva (45), in reparti chirurgici (9) e medici (29). L'età media dei pazienti era di $68 \pm 14$ anni.

I fattori di rischio maggiormente presenti furono: catetere urinario (presente nel $93 \%$ dei pazienti), terapia antibiotica $(89 \%)$, nutrizione parenterale $(55 \%)$, interventi chirurgici (47\%) e insufficienza renale (37\%). Essi sono compendiati in tabella 1 .

Il $28 \%$ dei pazienti presentava Candida spp. anche in altri distretti dell'organismo. In particolare 21/83 individui presentavano candidosi orofaringea o lieviti nell'espettorato e 2/48 donne candidosi vaginale. Candidemia fu riscontrata in 13 pazienti.

La candiduria era spesso associata a febbre $(61 \%$ dei pazienti) e ad infezioni batteriche (65\%). Nella maggior parte dei casi essa fu asintomatica ma il sedimento urinario presentava quasi costantemente leucociti.

Candida albicans fu la specie maggiormente isolata dalle urine (nel $61 \%$ dei pazienti), seguita da C. glabrata (18\%) e C. tropicalis (6\%); saltuariamente furono isolate altre specie (tabella 2). Tutti i pazienti presentarono la stessa specie nelle due urinocolture consecutive.

Candida albicans fu isolata dalle emocolture di dieci pazienti, C. glabrata da tre pazienti. Le stesse specie ritrovate nel sangue furono isolate nelle urine e, in sei pazienti, anche in altri campioni biologici (espettorato, tamponi orofaringei, tamponi vaginali).

Al $58 \%$ dei pazienti (48/83) fu somministrata una terapia antimicotica prevalentemente a base di fluconazolo (tabella 3) e ad alcuni di essi fu sostituito anche il catetere urinario. Gli altri 35 pazienti non furono sottoposti a terapia antimicotica (in 7 fu sostituito il catetere urinario).

I 13 pazienti con candiduria e candidemia furono tutti sottoposti a terapia antimicotica ad eccezione di uno (deceduto prima di iniziare il trattamento). L'evoluzione micologica (tabella 4) è stata investigata in 49 pazienti.

Per 38 di loro fu possibile evidenziare la risoluzione della candiduria che avvenne sia in presenza di terapia antifungina (31 pazienti) che in assenza ( 7 pazienti)

Per 42 pazienti è stato possibile fare diagnosi di candidosi urinaria accertata o probabile.

Data l'incompletezza di molte schede relative alla evoluzione micologica e alla diagnosi conclusiva non abbiamo potuto correlare questi dati con la terapia.

Tabella I. Alcuni fattori di rischio presenti in 83 pazienti

\begin{tabular}{lcc}
\hline Fattori di rischio & $\mathrm{N}^{\circ}$ pazienti & $\%$ \\
\hline Catetere urinario & 77 & 92,77 \\
\hline Terapia antibiotica & 74 & 89,16 \\
\hline Terapia corticosteroidea & 16 & 19,28 \\
\hline Terapia immunosoppressiva & 4 & 4,82 \\
\hline Nutrizione parenterale & 46 & 55,42 \\
\hline Interventi chirurgici & $39 *$ & 46,99 \\
\hline Insufficienza renale & $3 \mathrm{I}$ & 37,35 \\
\hline Patologie tumorali & 17 & 20,48 \\
\hline Ostruzione vie urinarie & 6 & 7,23 \\
\hline Procedure urologiche & 6 & 7,23 \\
\hline Neutropenia & 2 & 2,41 \\
\hline Candidemia & 13 & 15,66 \\
\hline Candida spp in altri distretti** & 23 & 27,71 \\
\hline
\end{tabular}

*8/39 a carico dell'apparato urinario;

**orofaringe, escreato, vagina

Tabella 2. Specie fungine isolate da urinocolture di 83 pazienti

\begin{tabular}{lcc} 
Specie fungina & $\mathrm{N}^{\circ}$ pazienti & $\%$ \\
\hline C. albicans & $5 \mathrm{I}$ & $6 \mathrm{I}, 45$ \\
\hline C. glabrata & 15 & 18,07 \\
\hline C. tropicalis & 5 & 6,02 \\
\hline C. parapsilosis & 3 & 3,61 \\
\hline C. kefyr & 2 & 2,41 \\
\hline C. dubliniensis & 1 & 1,20 \\
\hline C. krusei & $\mathrm{I}$ & 1,20 \\
\hline C. krusei + C. albicans & $\mathrm{I}$ & 1,20 \\
\hline C. lusitaniae & 1 & 1,20 \\
\hline Candida spp & 3 & 3,61
\end{tabular}


Tabella 3. Terapia antifungina somministrata a 48 pazienti. Antimicotico $\quad \mathrm{N}^{\circ}$ pazienti Fluconazolo $4 I^{*}$

Amfotericina B e.v.

Amfotericina B liposomiale

Amfotericina B (lav.vescicale)

Amfotericina B e.v.+ Fluconazolo

Amfotericina B (lav.vescicale) + Fluconazolo

altro

* I0 pazienti presentavano candiduria e candidemia

**I paziente presentava candiduria e candidemia

Tabella 4. Evoluzione micologica e diagnosi conclusiva Evoluzione micologica $\quad \mathrm{N}^{\circ}$ pazienti Risoluzione della candiduria sotto trattamento antifungino 31 Risoluzione della candiduria senza trattamento antifungino 7 Perdurare della candiduria sotto trattamento antifungino 9 Perdurare della candiduria senza trattamento antifungino 2 Non precisata 34

\section{Diagnosi conclusiva}

Micosi urinaria accertata/probabile

Micosi urinaria esclusa

Non precisata 42 3 38

\section{CONCLUSIONI}

Sebbene il campione analizzato, per l'esiguità dei dati, permetta di considerare come 'preliminari' i risultati ottenuti e, comunque, meritevoli di un ulteriore approfondimento, possiamo ipotizzare conclusioni significative:

1. - I pazienti che presentano candiduria sono in genere soggetti anziani e, preferibilmente, di sesso femminile. Fra i fattori favorenti risalta il posizionamento di cateteri urinari e la somministrazione di terapia antibiotica. Quest'ultima, specie se prolungata nel tempo, può infatti giustificare la modificazione dell'ecosistema microbico con la selezione di flora fungina abitualmente presente a livello del perineo o della vagina che può facilmente colonizzare/infettare le vie urinarie grazie alla presenza di cateteri urinari. La maggiore frequenza di candidurie nel sesso femminile è verosimilmente da porre in correlazione con questi motivi.

2. - La candiduria è frequente in pazienti sottoposti ad interventi chirurgici, a nutrizione parenterale e in presenza di insufficienza renale: tali eventi sono strettamente embricati tra loro, favorendo, insieme alle terapie antibiotiche ed ai cateterismi urinari, la costituzione di multifattoriali cause di rischio.

3. - Il $16 \%$ dei pazienti presentò candidemia. I dati non sono sufficienti per stabilire se la candidemia abbia determinato la localizzazione dei lieviti a livello urinario o se sia vero il contrario. D'altra parte, le due localizzazioni potrebbero essere indipendenti fra loro, anche se alcuni pazienti (6/13) risultavano colonizzati dalla stessa specie fungina anche in altri distretti dell'organismo. Caratterizzazioni intraspecifiche potrebbero stabilire se il ceppo isolato da escreato, orofaringe, vagina sia il medesimo ritrovato anche nelle emo- e nelle urinocolture.

4. - Candida albicans si è confermata come la causa prevalente di candidurie; tuttavia il ritrovamento di C. glabrata nel $18 \%$ dei pazienti mostra che questa specie è diventata sempre più frequente nella popolazione ospedalizzata probabilmente in relazione alla profilassi/terapia con fluconazolo ed alla selezione da questo prodotta con il conseguente emergere di stipiti resistenti $(1,6,8)$.

5. - La terapia antifungina non è stata effettuata nel $42 \%$ dei pazienti (per lo più asintomatici): la risoluzione micologica avuta in taluni casi conferma, d'altra parte, l'ipotesi di una contaminazione o colonizzazione solo transitoria.

Nei pazienti trattati, il fluconazolo è stato il farmaco di elezione, mentre solo raramente è stata utilizzata l'amfotericina $\mathrm{B}$ per via endovenosa o per lavaggi vescicali.

L'incompletezza dei dati relativi alla diagnosi conclusiva ed all'evoluzione micologica denota le difficoltà a porre diagnosi di candidosi urinaria e la scarsa abitudine dei clinici a richiedere controlli micologici nel tempo. E' indubbio che un attento e cadenzato monitoraggio microbiologico delle urine potrebbe apportare un valido aiuto alla diagnosi e potrebbe fornire maggiori conoscenze sull'evoluzione della candiduria in relazione al trattamento antimicotico.

\section{BIBLIOGRAFIA}

1. Al-Hedaithy SS, Fotedar R. Prevalence of Candida tropicalis in clinical specimens from patients with variable clinical syndromes over a 5-year period. Mycoses 1997; 40:111-3

2. Chabasse D. Intérêt de la numération des levures dans les urines. Revue de la littérature et résultats préliminaires d'une enquête multicentrique réalisée dans 15 centres hospitaliers universitaires. Ann Fr Anesth Reanim 2001; 20: 400-6

3. Faggi E, Farina C, Andreoni S, et al. Isolamento di lieviti dalle urine: un dilemma diagnostico e terapeutico. Proposta per un'indagine epidemiologica. Microbiologia Medica 2000; 15: 431-6.

4. Fisher JF, Newman CL, Sobel JD. Yeast in the urine: solutions for a budding problem. Clin Inf Dis 1995; 20: 183-9.

5. Gubbins PO, Scott A., McConnell, et al. Current management of funguria. Am J Health-Syst Pharm 1999; 56: 1929-1935

6. Harris AD, Castro J, Sheppard DC, et al. Risk factors for nosocomial candiduria due to Candida glabrata and Candida albicans. Clin Infect Dis 1999; 29: 9268.

7. Lundstrom T, Sobel J. Nosocomial candiduria: a review. Clin Infect Dis 2001; 32: 1602-7

8. Occhipinti DJ, Gubbins PO, Schreckenberger P, et al. 
Frequency, pathogenicity and microbiologic outcome of non Candida albicans candiduria. Eur J Microbiol Infect Dis 1994; 13: 459-67

9. Odds FC. Candidosis of the Urinary Tract. In Candida and Candidosis. A review and Bibliography. Bailliere Tindall Ed, London 1988; 169-74

10. Sobel JD, Kauffman CA, McKinsey D, et al. Candiduria: a randomized, double-blind study of treatment with fluconazole and placebo. Clin Infect Dis 2000; 30: 19-24

\section{Elisabetta Faggi}

Dipartimento di Sanità Pubblica

Sez Microbiologia, V.le Morgagni 48, 50134 Firenze

Tel 055-3262443; Fax 055-3262446

E-mail: efaggi@unifi.it 